\title{
Ab Initio Calculations on 1,2-Hydrogen Shifts in the Benzene Radical Cation and on Carbon Scrambling via an Isomerization to the Fulvene Structure
}

\author{
W. J. van der Hart \\ Leiden Institute of Chemistry, Gorlaeus Laboratories, Leiden University, Leiden, The Netherlands
}

Multireference configuration interaction (MRCI/ $/ 6-31 \mathrm{G}^{* *}$ ) ab initio calculations show that the barrier for hydrogen scrambling in the benzene radical cation is about $50 \mathrm{kcal} \mathrm{mol}^{-1}$. Once the internal energy is sufficient for a 1,2-hydrogen shift, the moving hydrogen can go to any position in the ring. The barrier for carbon scrambling via an isomerization to the fulvene structure is about $17 \mathrm{kcal} \mathrm{mol}^{-1}$ higher than that for hydrogen scrambling. Both of these values are far below the dissociation limit. (J Am Soc Mass Spectrom 1995, 6, 513-515)

A mong the classical problems in mass spectrometry is the carbon and hydrogen scrambling in the benzene radical cation and the probably connected observation that (collisionally induced) mass spectra of benzene and of many of its isomers show highly similar fragmentation patterns (see [1] for a review). In addition to this, studies of ion-molecule reactions of $\mathrm{C}_{6} \mathrm{H}_{6}$ radical cations from different neutral precursors [2-5] and of their photodissociation and/or charge exchange ionization mass spectra [6-8] show that, depending on the internal energy, these ions may isomerize to the benzene structure without decomposition.

In a recent article [9] from this laboratory it was shown that semiempirical calculations give a clear qualitative picture of the processes involved, which is in very good agreement with the experimental results. The conclusions from this work now are used as a starting point for $a b$ initio calculations. In this article we report the results for the first processes studied: hydrogen scrambling via consecutive 1,2-hydrogen shifts and carbon scrambling via an isomerization to the fulvene structure. Ab initio calculations on the 1,2hydrogen shift in the benzene radical cation were published previously by Gallup et al. [10], who obtained a value of $1.86 \mathrm{eV}$ for the energy difference between the benzene radical cation and the product ion (structure 2 in Scheme I). This value is significantly below the dissociation limit of $3.88 \mathrm{eV}$ [11]. In the present article it will be shown that the other barriers involved also are far below the dissociation threshold.

Address reprint requests to Dr. W. J. van der Hart, Leiden Institute of Chemistry, Leiden University, P. O. Box 9502, 2300 RA Leiden, The Netherlands.

\section{Methods}

$\mathrm{Ab}$ initio calculations via the $6-31 \mathrm{G}^{* *}$ basis set were performed with both the GAMESS-UK [12] and the Gaussian 92 [13] program packages. Stable ion structures and transition states were optimized without symmetry at the (restricted) self-consistent field (SCF) level and tested by a calculation of the vibrational frequencies. For these optimized structures, multireference configuration interaction (MRCI) calculations with single and double excitations were done with the Table CI ([14] and references cited therein) option of GAMESS-UK. In these calculations the lowest 10 occupied and the highest 40 virtual molecular orbitals were discarded. All configurations that had a coefficient squared higher than 0.0025 in the final ground state wave function or higher than 0.0030 in the wavefunction for the second root (of the same symmetry) were used as reference configurations. The extrapolation threshold was set at the lowest value that was compatible with the maximum number of 30,000 configurations in the final diagonalization. For the benzene radical cation and all transition states, the threshold was $5 \mu$ hartree; for the remaining stable ion structures it was $7.5 \mu$ hartree (see Table 1). The MRCI values given in Table 1 are the estimated full configuration interaction $(\mathrm{CI})$ values including a size-consistency correction. The MRCI values in Table 2 are relative to the benzene radical cation energy calculated with the same extrapolation threshold.

\section{Results and Discussion}

The processes involved are shown in Scheme I and the results of the calculations, together with the modified 
Table 1. Energies in hartree (more detailed results can be obtained from the author)

\begin{tabular}{lccc}
\hline & SCF & MRCI & $\begin{array}{c}\text { Zero point energy } \\
\text { (SCF) }\end{array}$ \\
\hline \hline Benzene 1 & -230.421222 & $-230.868588^{\mathrm{b}}$ & 0.105319 \\
Benzene & & $-230.870064^{\mathrm{c}}$ & \\
$T_{1}$ & -230.337993 & $-230.786505^{\mathrm{c}}$ & 0.101732 \\
$\mathbf{2}$ & -230.369397 & $-230.812758^{\mathrm{b}}$ & 0.104552 \\
$T_{2}$ & -230.336971 & $-230.786555^{\mathrm{c}}$ & 0.102068 \\
$\mathbf{3}$ & -230.365100 & $-230.812943^{\mathrm{b}} \cdot$ & 0.104318 \\
$T_{3}$ & -230.339736 & $-230.790783^{\mathrm{c}}$ & 0.102059 \\
$\mathbf{4}$ & -230.371634 & $-230.810205^{\mathrm{b}}$ & 0.104389 \\
$T_{4}$ & -230.303401 & $-230.760790^{\mathrm{c}}$ & 0.102741 \\
$\mathbf{5}$ & -230.328165 & $-230.778472^{\mathrm{c}}$ & 0.104709 \\
$T_{5}$ & -230.322075 & $-230.787985^{\mathrm{c}}$ & 0.102744 \\
Fulvene 6 & -230.401340 & $-230.849193^{\mathrm{b}}$ & 0.104267 \\
\hline
\end{tabular}

Unscaled value.

Extrapolation threshold is $7.5 \mu$ hartree.

'Extrapolation threshold is $5.0 \mu$ hartree.

neglect of differential overlap (MNDO) results from [9], are given in Tables 1 and 2.

From the results it is first of all clear that the semiempirical values from [9] for the energy differences between the stable ion structures are in reasonable agreement with the $a b$ initio results. Also the relative semiempirical values for the isomerization barriers are more or less correct, but their absolute values are systematically some $10-20 \mathrm{kcal} \mathrm{mol}^{-1}$ too high.

Structures 2, 3, and 4, obtained from the benzene structure by consecutive 1,2-hydrogen shifts, have essentially identical heats of formation of about $35 \mathrm{kcal}$ $\mathrm{mol}^{-1}$, which is $1.52 \mathrm{eV}$ higher than that of the benzene radical cation, which is $8 \mathrm{kcal} \mathrm{mol}^{-1}$ lower than the value given in [10]. For all three barriers that connect the benzene structure and structures 2,3 , and 4, a value of about $50 \mathrm{kcal} \mathrm{mol}^{-1}$ is obtained, and the value for $T_{3}$ is slightly lower than that for the other two barriers. It thus follows that once the internal energy of the benzene radical cation is sufficient for a 1,2-hydrogen shift, the moving hydrogen atom can go to any position in the benzene ring. This event leads to much faster hydrogen scrambling than follows from

Table 2. Relative energies in kilocalories per mole. The ab initio values include the SCF zero point energy scaled by a factor of 0.89

\begin{tabular}{lccc}
\hline & MNDO [9] & SCF & MRCl \\
\hline \hline Benzene 1 & 0 & 0 & 0 \\
$T_{1}$ & 72 & 50.2 & 50.4 \\
Structure 2 & 39 & 32.1 & 34.6 \\
$T_{2}$ & 73 & 51.1 & 50.6 \\
Structure 3 & 40 & 34.6 & 34.3 \\
$T_{3}$ & 72 & 49.3 & 47.9 \\
Structure 4 & 39 & 30.6 & 36.1 \\
$T_{4}$ & 78 & 72.5 & 67.2 \\
Intermediate 5 & 68 & 58.1 & 57.2 \\
$T_{5}$ & 71 & 60.8 & 50.1 \\
Fulvene 6 & 13 & 11.9 & 11.6 \\
\hline
\end{tabular}
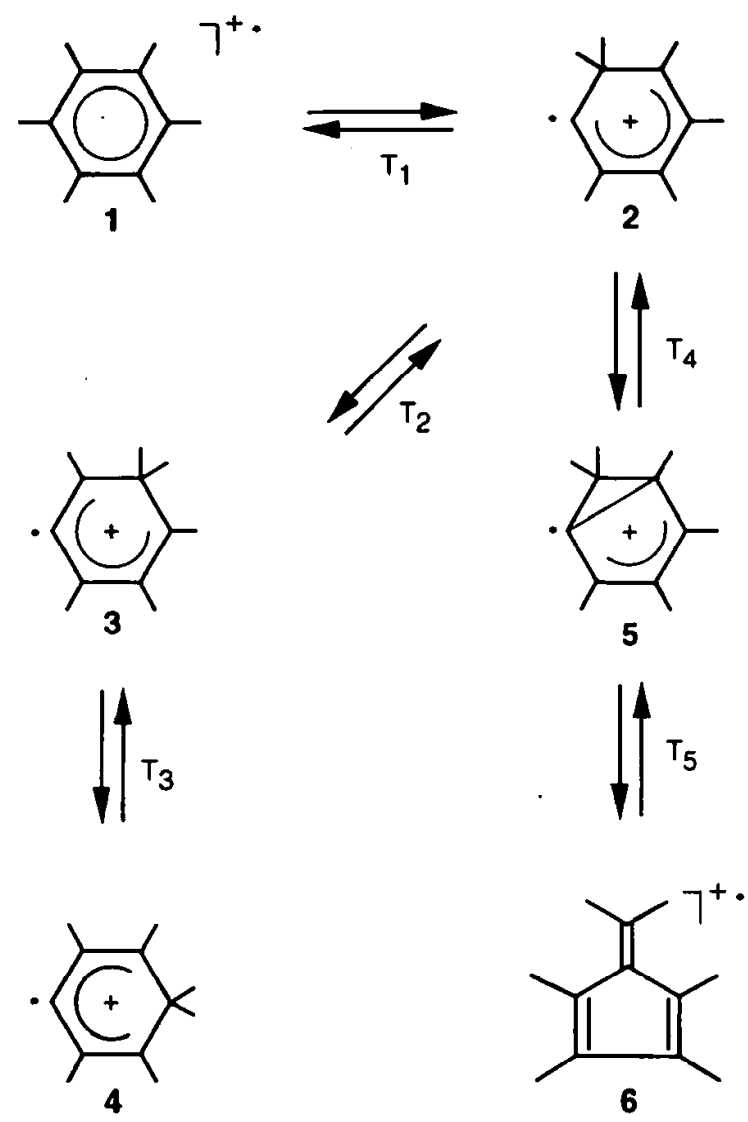

Scheme I

[10], where only structure 2 was considered to be an intermediate structure in hydrogen scrambling.

The barrier for carbon scrambling by an isomerization of structure 2 to the fulvene structure is approximately $17 \mathrm{kcal} \mathrm{mol}^{-1}$ higher than that for hydrogen scrambling. This observation gives a simple explanation for the well-known independence of hydrogen and carbon scrambling in the benzene radical cation [1]. Interestingly, both the semiempirical and the $a b$ initio SCF calculations suggest that the isomerization of structure 2 to the fulvene structure proceeds via the stable intermediate 5 . The MRCI results, however, show that this intermediate very probably does not exist. Both the barriers for hydrogen scrambling $(50.6 \mathrm{kcal}$ $\left.\mathrm{mol}^{-1}=2.19 \mathrm{eV}\right)$ and that for carbon scrambling $(67.2$ kcal $\mathrm{mol}^{-1}=2.91 \mathrm{eV}$ ) are significantly below the dissociation threshold of $3.88 \mathrm{eV}$.

According to the semiempirical results in [9], many isomerization reactions between classical and nonclassical $\mathrm{C}_{6} \mathrm{H}_{6}$ radical cation structures have barriers below the dissociation limit. At higher internal energies, many of these reactions may contribute to hydrogen scrambling in $\mathrm{C}_{6} \mathrm{H}_{6}$ radical cations and some to carbon scrambling. The calculations indicate that the lowest barriers that can be reached directly from the structures in Scheme I are those for ring opening of structure 3 to the 1,3-hexadien-5-yne structure and for ring contraction of both structures 2 and 3 to nonclassical ion structures with a five-membered ring and an exo- 
cyclic $\mathrm{CH}$ bond, and from these latter structures to the 1,3-hexadien-5-yne structure (see Schemes 4 and 7 in [9]). These reactions, with estimated barriers [9] some 5-10 $\mathrm{kcal} \mathrm{mol}^{-1}$ above the barrier for isomerization of benzene radical cations to the fulvene structure, will be the subject of further ab initio calculations.

\section{Acknowledgment}

The author wishes to thank Dr. M. C. van Hemert for very helpful advice on the use of Table $\mathrm{CI}$.

\section{References}

1. Rosenstock, H. M.; Dannacher, J.; Liebman, J. Radiat. Phys. Chem. 1982, 20, 7.

2. Gross, M. L.; Russell, D. H.; Aerni, R. J.; Bronczyk, S. A. J. Am. Chem. Soc. 1977, 99, 3603.

3. Russell, D. H.; Gross, M. L. I. Am. Chem. Soc. 1980, 102, 6279.

4. Miller, D. L.; Gross, M. L. J. Am. Chem. Soc. 1983, 105, 3783.

5. Miller, D. L.; Gross, M. L. J. Am. Chem. Soc. 1983, 105, 4239.
6. van der Hart, W. J.; de Koning, L. J.; Nibbering, N. M. M.; Gross, M. L. Int. J. Mass Spectrom. Ion Processes 1986, 72, 99.

7. van de Guchte, W. J.; van der Hart, W. J. Int. J. Mass Spectrom. Ion Processes 1993, 123, 7.

8. van de Guchte, W. J.; van der Hart, W. J.; de Koning, L. J.; Nibbering, N. M. M.; Dunbar, R. C. Int. J. Mass Spectrom. Ion Processes 1993, 123, 11.

9. van der Hart, W. J. Int. J. Mass Spectrom. Ion Processes 1994, 130, 173.

10. Gallup, G. A.; Steinheider, D.; Gross, M. L. Int. J. Mass Spectrom. Ion Phys. 1976, 22, 185.

11. Klippenstein, S. J.; Faulk, J. D.; Dunbar, R. C. I. Chem. Phys. 1993, 98, 243.

12. Guest, M. F.; Fantucci, P.; Harrison, R. J.; Kendrick, J.; van Lenthe, J. H.; Schoeffel, K.; Sherwood, P. GAMESS-UK User's Guide and Reference Manual, Revision C.0; Computing for Science (CFS) Ltd.: Daresbury Laboratory, Daresbury, UK, 1992.

13. Frisch, M. J.; Trucks, G. W.; Head-Gordon, M.; Gill, P. M. W.; Wong, M. W.; Foresman, J. B.; Johnson, B. G.; Schlegel, H. B.; Robb, M. A.; Replogle, E. S; Gomperts, R.; Andres, J. L.; Raghavachari, K.; Binkley, J. S.; Gonzalez, C.; Martin, R. L.; Fox, D. J.; Defrees, D. J.; Baker, J.; Stewart, J. J. P.; Pople, J. A. Gaussian 92, Revision E.2; Gaussian, Inc.: Pittsburgh PA, 1992. 14. Buenker, R. J.; Phillips, R. A. J. Mol. Struct. 1985, 123, 291. 